\title{
Dynamic Discrete GM $(1,1)$ Model and Its Application in the Prediction of Urbanization Conflict Events
}

\author{
Ersi Liu, Qiangqiang Wang, Xinran Ge, and Wei Zhou \\ International Business School, Yunnan University of Finance and Economics, Kunming 650221, China \\ Correspondence should be addressed to Qiangqiang Wang; wqq1801@163.com
}

Received 23 March 2016; Accepted 16 May 2016

Academic Editor: Juan R. Torregrosa

Copyright (C) 2016 Ersi Liu et al. This is an open access article distributed under the Creative Commons Attribution License, which permits unrestricted use, distribution, and reproduction in any medium, provided the original work is properly cited.

In the empirical researches, the discrete GM $(1,1)$ model is not always fitted well, and sometimes the forecasting error is large. In order to solve this issue, this study proposes a dynamic discrete GM $(1,1)$ model based on the grey prediction theory and the GM $(1,1)$ model. In this paper, we use the equal division technology to fit the concavity and convexity of the cumulative sequence and then construct two dynamic average values. Based on the dynamic average values, we further develop two dynamic discrete GM $(1,1)$ models and provide the gradual heuristics method to draw the initial equal division number and the dichotomy approach to optimize the equal division number. Finally, based on an empirical analysis of the number of conflict events in the urbanization process in China, we verify that the dynamic discrete GM $(1,1)$ model has higher fitting and prediction accuracy than the GM $(1,1)$ model and the discrete GM $(1,1)$ model, and its prediction result is beneficial to the government for prevention and solution of the urbanization conflict events.

\section{Introduction}

After years of development $[1,2]$, the grey system theory has formed a relatively complete system theory. The GM $(1,1)$ model, with its unique advantages, is widely used in several fields such as economics, management, industry, agriculture, military, science, and education. The obvious advantage of the GM $(1,1)$ model is that the model can be used to model and predict a small amount of sequence data.

Further, the optimization of the grey model and the improvement of the grey system theory have acquired a lot of achievements. In the research area of discrete GM $(1,1)$ models, Xie and Liu [3] proposed an unbiased discrete GM $(1,1)$ model by focusing on the forecast stability problem of the GM $(1,1)$ model and analyzing its causes in detail, from continuous to discrete. Xie and Liu [4] outlined three forms of discrete GM $(1,1)$ model; meanwhile, they also proposed a method for approximately fitting the homogeneous exponent. Yao and Liu [5] studied the nonlinear and nonnegative sequence fitting issue and then proposed an improved discrete GM $(1,1)$ model. Wang et al. [6] proved that a discrete homogeneous exponent calculated by once accumulated operation can be transformed into a discrete nonhomogeneous exponent. Based on that, they further constructed an optimized discrete GM $(1,1)$ model based on an improved background value. Yao et al. [7] studied parameter properties of the new information discrete GM $(1,1)$ model and proposed the piecewise modified new information discrete GM $(1,1)$ model and analyzed its modeling mechanism. To further improve the GM $(1,1)$ model and the discrete GM $(1,1)$ model, Zhou et al. [8] proposed the generalized GM $(1,1)$ model based on an optimal stepwise ratio, which can effectively include the GM $(1,1)$ model and the discrete GM (1,1) model; Kayacan et al. [9] analyzed the advantages and disadvantages of the discrete GM $(1,1)$ model in the least square method and then gave a new sequence discrete GM $(1,1)$ model based on the structural risk minimization; Li et al. [10] used the two-step method to construct a hybrid model by combining the GM $(1,1)$ model and the discrete GM $(1,1)$ model; Zhou and He [11] proposed a new generalized GM $(1,1)$ model which can be used to depict the discrete GM $(1,1)$ model and optimized it by using four kinds of methods to obtain better fitting prediction results; 
Liu et al. [12] developed a new improved discrete GM (1,1) model by introducing the neural network. Yang and Zhao [13] corrected the fixed error of the GM $(1,1)$ power model and then gave a discrete GM $(1,1)$ power model. Recently, Zhou and Zhang [14] identified the prediction inaccuracies in the GM $(1,1)$ model and the discrete GM $(1,1)$ model based on the presence of singularities in the real small sample data and then proposed the improved metabolism GM $(1,1)$ model. The aforementioned models belong to the discrete GM $(1,1)$ model and provide the basis for the new discrete GM $(1,1)$ model proposed in this paper.

Moreover, another key issue of this paper is the research of urbanization conflict. Many studies have been taken which can be reviewed as follows: Portes and Roberts [15] tested the level of urban development of the Latin American cities in recent decades based on comparable data from six countries. $\mathrm{Yu}$ et al. [16] used the brainstorming and semistructured interview to identify urbanization conflict risk factors and applied them in the rapid urbanization process in China. Yu et al. [17] further studied the conflict about society, economics, and the environment in the process of urbanization. Zhou et al. [18] used Hangzhou Bay as the object of research to design a set of methods to optimize the quality of urbanization, which is conducive to improving the quality of urbanization and reducing the conflict associated with urbanization. Su et al. [19] studied the acceleration of the urbanization process, due to which China's urban and rural income gap continues to expand and cause certain conflict problems. Wang et al. [20] studied China's urbanization process since 1990, which brought rapid economic growth; however, it also created a series of problems. Recently, Wang et al. [20] defined the levels of urban and rural development and the transformation of urban and rural coordination as three indicators to evaluate urbanization, with the objective of trying to improve the quality of urbanization from the aspects and reduce urban-rural conflict. However, as an important issue in the urbanization conflict, the data analysis and prediction have not been studied fully.

In the context of the abovementioned issues, this paper proposes a new discrete GM $(1,1)$ model, namely, the dynamic discrete GM $(1,1)$ model, to predict the number of the urbanization conflict events in China. To do this, this paper develops two forms of the dynamic discrete GM $(1,1)$ model which can be used to analyze realistic data that is either increasing or decreasing and propose two series of dynamic average values with an equal division technology. For the development coefficient and grey action parameter in the new grey models, this paper calculates them by combining the trapezoidal background formula with the least square method. Further, this paper develops heuristics and the method of valuing the equal division number. In recent years, group conflict occurred progressively which resulted from the acceleration of the urbanization process in China. Thus, this paper forecasts the number of urbanization conflict events in China based on the proposed dynamic discrete GM $(1,1)$ model. The results show that the proposed model can be effectively used in this filed.

In order to do that, this paper is organized as follows: the discrete GM $(1,1)$ model and its background value are analyzed in Section 2. Based on these, the convex and concave dynamic discrete GM $(1,1)$ models are proposed and their properties are discussed in Section 3. In Section 4, the solution and optimization methods of the equal division number are analyzed. In Section 5, a practical example is provided to illustrate the proposed grey models. The paper ends with conclusions in Section 6.

\section{Analysis of the Discrete GM $(1,1)$ Model and the Background Value}

The discrete GM $(1,1)$ model is an important part of the grey system theory. This paper first analyzes the discrete GM $(1,1)$ model proposed by Xie and Liu [3] and then studies its model calculation and construction process, based on which we provide the foundation for further dynamic discrete model construction.

Let a nonnegative small sample sequence $X^{(0)}=$ $\left(x^{(0)}(1), x^{(0)}(2), \ldots, x^{(0)}(n)\right)$ be the original data sequence, $x^{(0)}(k) \geq 0$ and $k=1,2, \ldots, n$. As a small sample sequence, $n$ is generally small at $n \leq 10$. Then, $X^{(1)}$ is called the once accumulated generating operation (1-AGO) sequence of $X^{(0)}$, where $X^{(1)}=\left(x^{(1)}(1), x^{(1)}(2), \ldots, x^{(1)}(n)\right)$ and

$$
x^{(1)}(k)=\sum_{i=1}^{k} x^{(0)}(i), \quad k=1,2, \ldots, n
$$

Definition 1. The original form of the GM $(1,1)$ model is defined as

$$
x^{(0)}(k)+a x^{(1)}(k)=b,
$$

where $x^{(0)}(k) \geq 0$ is the original data, $x^{(1)}(k)$ is the 1-AGO data, $-a$ is the developing coefficient, and $b$ is the grey action parameter.

Definition 2. The average value form of GM $(1,1)$ model is defined as

$$
x^{(0)}(k)+a z^{(1)}(k)=b \text {, }
$$

where $x^{(0)}(k) \geq 0$ is the original data, $z^{(1)}(k)$ is the average value satisfied $z^{(1)}(k)=0.5 \cdot\left(z^{(1)}(k-1)+z^{(1)}(k)\right),-a$ is the developing coefficient, and $b$ is the grey action parameter.

The original and average value forms of the GM $(1,1)$ model are two difference equations, namely, (2) and (3), which can be estimated by the least square method. Their parameters estimated by the least square method satisfy the following condition:

$$
\widehat{\alpha}=\left(B^{T} B\right)^{-1} B^{T} Y,
$$


where $\widehat{\alpha}=(a, b)^{T}$ and

$$
\begin{aligned}
& Y=\left[\begin{array}{c}
x^{(0)}(2) \\
x^{(0)}(3) \\
\ldots \\
x^{(0)}(n)
\end{array}\right], \\
& B=\left[\begin{array}{cc}
-z^{(1)}(2) & 1 \\
-z^{(1)}(3) & 1 \\
\ldots & \ldots \\
-z^{(1)}(n) & 1
\end{array}\right] .
\end{aligned}
$$

Thus, we can obtain the parameters $a$ and $b$.

To improve the GM $(1,1)$ model, many studies are presented to broaden the scope of the background value and improve its effectiveness. For example, Tan [21] developed a new method to construct the background value, namely, using the piecewise method. The fitting approaches in Luo et al. [22] and Liu et al. [23] are based on the use of the homogeneous exponent function and are applied to fit the cumulative sequence, which can be used to calculate the optimal background value. Based on this, $b / a$ was fully considered in Liu et al. [23], and a new background value optimization formula was provided. Tang and Xiang [24] and Wang and Yang [25] used the two interpolation methods and the combination interpolation method to fit the background value, respectively; however, the calculation in both papers is complex. Moreover, Wang et al. [6] developed a generalized background value which can be used to depict other background values and presented as follows.

Definition 3 (see [6]). An optimal background value is defined as

$$
\begin{aligned}
Z^{(1)}(k)= & \frac{x^{(0)}(k)}{\ln x^{(0)}(k)-\ln x^{(0)}(k-1)} \\
& +\frac{\left[x^{(0)}(k-1)\right]^{k}}{\left[x^{(0)}(k)\right]^{k-2}\left[x^{(0)}(k-1)-x^{(0)}(k)\right]},
\end{aligned}
$$

where $x^{(0)}(k) \geq 0$ is the original data, $z^{(1)}(k)$ is the average value calculated from the complete homogeneous original data, and $k=1,2, \ldots, n$.

This background value can be combined with (6) to construct the optimal GM $(1,1)$ model, which is called OGM $(1,1)$ model.

Further, we introduce the discrete GM $(1,1)$ model and analyze it as follows.

Definition 4 (see [3]). The discrete GM (1,1) model, namely, DGM $(1,1)$ model, is defined as

$$
x^{(1)}(k+1)=\beta_{1} x^{(1)}(k)+\beta_{2},
$$

where $x^{(1)}(k)$ is the 1-AGO data, $\beta_{1}$ is the developing coefficient, and $\beta_{2}$ is the grey action parameter.
In (7), if we substitute its original data and 1-AGO data, that is, $x^{(0)}(k+1)=x^{(1)}(k+1)-x^{(1)}(k)$ and $z^{(1)}(k+1)=$ $0.5 x^{(1)}(k+1)+0.5 x^{(1)}(k)$, into the average value forms of the GM $(1,1)$ model (see (3)), we can get

$$
x^{(1)}(k+1)-x^{(1)}(k)+a\left(\frac{x^{(1)}(k+1)+x^{(1)}(k)}{2}\right)
$$

$$
=b \text {, }
$$

$$
x^{(1)}(k+1)=\left(\frac{1-0.5 a}{1+0.5 a}\right) x^{(1)}(k)+\frac{b}{1+0.5 a} .
$$

Let $\beta_{1}=((1-0.5 a) /(1+0.5 a))$ and $\beta_{2}=b /(1+0.5 a)$; then (9) can be obtained.

According to the least square method, the parameter vector in (4) can be obtained. Here, $z^{(1)}(k)$ in (3) is the background value. By analyzing the structure of the discrete GM $(1,1)$ model, it can be found that the form of the average value determines the form of the model and the precision of the model is determined by the accuracy of the average value. Therefore, in the following section, we use the segmentation method referred to in Tan [21] to develop a dynamic discrete GM $(1,1)$ model to depict the index data, which will be better than the GM $(1,1)$ model and the discrete GM $(1,1)$ model.

\section{Construction of the Dynamic Discrete GM $(1,1)$ Model}

In this section, we construct the convex and concave dynamic discrete GM $(1,1)$ models based on a generalized dynamic average value. Then, we analyze some attractive properties of the dynamic discrete GM $(1,1)$ models.

3.1. Construction of the Dynamic Average Value. The constructions of the average value and the background value are similar. They can be explained as follows: to construct a new average value (or a background value) by using the method of constructing the background value proposed in Tan [21]. The key point is that $[k, k+1]$ is divided into several rectangles equally, and the area of the curved surface is replaced by the area of the rectangles. Theoretically, there is a value of $n$ (may not be the integer) that will make the area of $n$ rectangles equal to the area of the curved surface. Then, in the process of constructing the new average value by using the equal division technology, we find two kinds of states: the original data increment and the original data decrement. The once accumulated sequence of the original data increment is a concave function (see Figure 1), and the once accumulated sequence of the original data decrement is a convex function (see Figure 2).

For a sequence with different functional characteristics, the processes of average value construction are not the same. The specific steps are as follows.

Step 1. The interval of the once accumulated sequence $[k, k+$ $1]$ is divided into $n$ equal parts, and the length of every part is $1 / n$; then we can constitute $n$ small rectangles. 


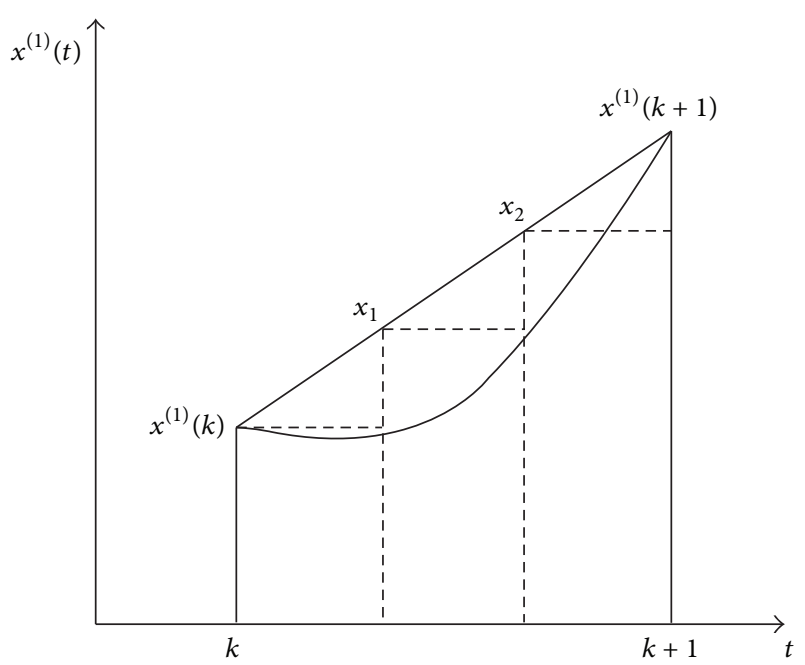

Figure 1: The concave once accumulated sequence.

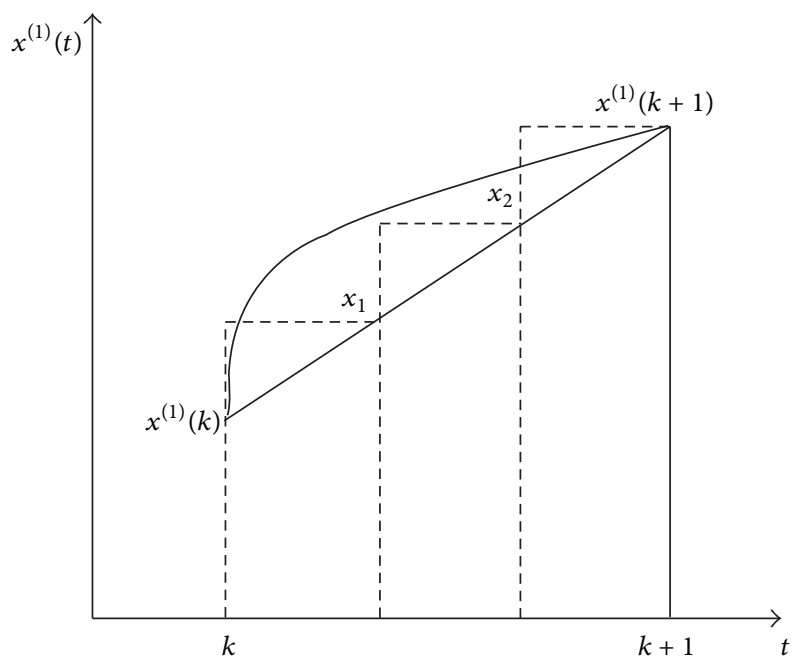

Figure 2: The convex once accumulated sequence.

Step 2. Calculate the height of first small rectangle by the following methods. When the once accumulated sequence has the characteristics of a concave function, $x^{(1)}(k)$ is regarded as the height of first small rectangle and $\left(x^{(1)}(k+1)-\right.$ $\left.x^{(1)}(k)\right) / n$ is increased in turn; when the once accumulated sequence has the properties of a convex function, $x^{(1)}(k+1)$ is considered to be the height of first small rectangle and then reduces $\left(x^{(1)}(k+1)-x^{(1)}(k)\right) / n$ in order. Thus, we can obtain the height of all small rectangles.

Step 3. Calculate the sum of the areas of the small rectangles which is regarded as the average value as follows. The height obtained from Step 2 is multiplied by the equal width, and then we can obtain the area of each small rectangle. Then through the summation we can get the area of the curved surface of the once accumulated sequence, which is the average value.
Theorem 5. When the once accumulated sequence has the characteristic of the convex function, based on the equal division technology, the dynamic average value can be presented as

$$
\begin{aligned}
t_{n}^{(1)} & (k+1) \\
& =\frac{1}{2 n}\left[(n-1) x^{(1)}(k)+(n+1) x^{(1)}(k+1)\right],
\end{aligned}
$$

where $x^{(1)}(k)$ is the once accumulated data of $k, x^{(1)}(k+1)$ is the once accumulated data of $k+1, n$ is the equal division number of the interval $[k, k+1]$, and $n$ represents the equal division number of $t_{n}^{(1)}(k+1)$.

Proof. As shown in Figure 2, if the equal division number is $n$, then we can get

$$
\begin{array}{r}
x_{i}=x^{(1)}(k+1)-\frac{n-i}{n}\left(x^{(1)}(k+1)-x^{(1)}(k)\right), \\
\quad i=1,2, n-1,
\end{array}
$$

where $x_{i}$ is the following $i$ point of $x^{(1)}(k)$.

By calculating the sum of the areas of the small rectangles, we have

$$
\begin{aligned}
S_{n} & =\frac{1}{n}\left(x^{(1)}(k+1)+\sum_{i=1}^{n-1} x_{i}\right) \\
& =\frac{1}{2 n}\left[(n-1) x^{(1)}(k)+(n+1) x^{(1)}(k+1)\right] .
\end{aligned}
$$

Therefore, the average value of the convex once accumulated sequence in the discrete GM $(1,1)$ model is

$$
\begin{aligned}
t_{n}^{(1)} & (k+1) \\
& =\frac{1}{2 n}\left[(n-1) x^{(1)}(k)+(n+1) x^{(1)}(k+1)\right] .
\end{aligned}
$$

Theorem 6 (see [21]). If the once accumulated sequence has the characteristic of the concave function, based on the equal division technology, the dynamic average value can be presented as

$$
\begin{aligned}
t_{n}^{(1)} & (k+1) \\
\quad & \frac{1}{2 n}\left[(n-1) x^{(1)}(k+1)+(n+1) x^{(1)}(k)\right],
\end{aligned}
$$

where $x^{(1)}(k)$ is the once accumulated data of $k, x^{(1)}(k+1)$ is the once accumulated data of $k+1, n$ is the equal division number of the interval $[k, k+1]$, and $n$ represents the equal division number of $t_{n}^{(1)}(k+1)$.

Based on Theorems 5 and 6, the following definitions are provided. 
Definition 7. The convex average value is defined as

$$
\begin{aligned}
t_{n}^{(1)} & (k+1)=S_{n} \\
& =\frac{1}{2 n}\left[(n-1) x^{(1)}(k)+(n+1) x^{(1)}(k+1)\right],
\end{aligned}
$$

where $t_{n}^{(1)}(k+1)$ is the convex average value, $x^{(1)}(k)$ is the 1 -AGO data, and $k=1,2, \ldots, n$.

Definition 8. The concave average value is defined as

$$
\begin{aligned}
p_{n}^{(1)} & (k+1)=S_{n} \\
& =\frac{1}{2 n}\left[(n-1) x^{(1)}(k+1)+(n+1) x^{(1)}(k)\right],
\end{aligned}
$$

where $p_{n}^{(1)}(k+1)$ is the concave average value, $x^{(1)}(k)$ is the 1 -AGO data, and $k=1,2, \ldots, n$.

Both the convex average value and the concave average value are dynamic average values of the equal division number $n$ and can be used in the dynamic discrete GM $(1,1)$ model.

3.2. The Concave and Convex Dynamic Discrete GM $(1,1)$ Models. According to Theorems 5 and 6, it is found that the two kinds of the average values mentioned above are dynamic with equal division interval. As compared to the previous expression of the average, the two values have more extensive adaptability and can fit the GM $(1,1)$ model better. On the basis of the above definitions and theories, two new discrete grey models, namely, the convex and concave dynamic discrete GM $(1,1)$ models, are proposed as follows.

Definition 9. The concave dynamic discrete GM $(1,1)$ model is defined as

$$
x^{(1)}(k+1)=\frac{2 n-a(n+1)}{2 n+a(n-1)} x^{(1)}(k)+\frac{2 n b}{2 n+a(n-1)},
$$

where $x^{(1)}(k)$ is the 1-AGO data, $-a$ is the developing coefficient, $b$ is the grey action parameter, $x^{(1)}(k)$ is the once accumulated data of $k, x^{(1)}(k+1)$ is the once accumulated data of $k+1$, and $n$ is the equal division number.

Definition 10. The convex dynamic discrete GM $(1,1)$ model is defined as

$$
x^{(1)}(k+1)=\frac{2 n-a(n-1)}{2 n+a(n+1)} x^{(1)}(k)+\frac{2 n b}{2 n+a(n+1)},
$$

where $x^{(1)}(k)$ is the 1-AGO data, $-a$ is the developing coefficient, $b$ is the grey action parameter, $x^{(1)}(k)$ is the once accumulated data of $k, x^{(1)}(k+1)$ is the once accumulated data of $k+1$, and $n$ is the equal division number.
Theorem 11. The restored function of the convex dynamic discrete GM $(1,1)$ model can be presented as follows:

$$
\begin{aligned}
\hat{x}^{(0)}(k+1)= & \left(\frac{-2 n a}{2 n-a(n-1)}\right)\left(x^{(0)}(1)-\frac{b}{a}\right) \\
& \cdot\left(\frac{2 n-a(n-1)}{2 n+a(n+1)}\right)^{k},
\end{aligned}
$$

where $a$ is the development coefficient, $b$ is the grey action parameter, $x^{(0)}(1)$ is the first original data, $\widehat{x}^{(0)}(k+1)$ is the simulated value of original data of $k+1$, and $n$ is the equal division number of the interval $[k, k+1]$.

Proof. If we substitute $x^{(0)}(k+1)=x^{(1)}(k+1)-x^{(1)}(k)$ and $t_{n}^{(1)}(k+1)=\left[(n-1) x^{(1)}(k)+(n+1) x^{(1)}(k+1)\right] / 2 n$ into the average value form of the GM $(1,1)$ model, then we can get the convex dynamic discrete GM $(1,1)$ model including the equal division number $n$ as follows:

$$
\begin{aligned}
& x^{(1)}(k+1)-x^{(1)}(k) \\
& \quad+\frac{a}{2 n}\left[(n+1) x^{(1)}(k+1)+(n-1) x^{(1)}(k)\right] \\
& =b .
\end{aligned}
$$

Based on the computation method of the difference equation, we can calculate the time responded function, and then the restored function can be obtained as

$$
\begin{aligned}
\widehat{x}^{(0)}(k+1)= & \left(\frac{-2 n a}{2 n-a(n-1)}\right)\left(x^{(0)}(1)-\frac{b}{a}\right) \\
& \cdot\left(\frac{2 n-a(n-1)}{2 n+a(n+1)}\right)^{k} .
\end{aligned}
$$

Thus, we complete the proof of Theorem 11.

Theorem 12. The restored function of the concave dynamic discrete GM $(1,1)$ model can be presented as follows:

$$
\begin{aligned}
\hat{x}^{(0)}(k+1)= & \left(\frac{-2 n a}{2 n-a(n+1)}\right)\left(x^{(0)}(1)-\frac{b}{a}\right) \\
& \cdot\left(\frac{2 n-a(n+1)}{2 n+a(n-1)}\right)^{k},
\end{aligned}
$$

where $a$ is the development coefficient, $b$ is the grey action parameter, $x^{(0)}(1)$ is the first original data, $\hat{x}^{(0)}(k+1)$ is the simulated value of original data of $k+1$, and $n$ is the equal division number of the interval $[k, k+1]$.

Proof. Similar to the proof of Theorem 11, Theorem 12 can be obtained easily.

These two types of dynamic discrete GM $(1,1)$ model constitute the complete dynamic discrete GM (1,1) model. The concave dynamic discrete GM $(1,1)$ model, the convex dynamic discrete GM $(1,1)$ model, and their restored functions are the dynamic function of the equal division number $n$. Furthermore, some desired properties of the two dynamic discrete GM $(1,1)$ models are analyzed in the next subsection. 
3.3. Some Properties of the Dynamic Discrete GM $(1,1)$ Models. The above dynamic discrete GM $(1,1)$ models are developed by introducing the improved average value. Therefore, they not only have original properties of the discrete GM $(1,1)$ model but also have the following special characteristics.

Property 1 (homogeneous exponential property). The restored functions of the concave dynamic discrete GM $(1,1)$ model and the convex dynamic discrete GM $(1,1)$ model are homogeneous exponential functions.

Proof. (1) According to the restored function of the concave dynamic discrete GM $(1,1)$ model, we can get

$$
\begin{aligned}
\sigma(k)=\frac{\widehat{x}^{(0)}(k+1)}{\widehat{x}^{(0)}(k)}=\frac{2 n-a(n+1)}{2 n+a(n-1)} & =\text { const, } \\
& k=1,2, \ldots, N .
\end{aligned}
$$

(2) According to the restored function of the convex dynamic discrete GM $(1,1)$ model, we can get

$$
\begin{aligned}
\sigma(k)=\frac{\hat{x}^{(0)}(k+1)}{\hat{x}^{(0)}(k)}=\frac{2 n-a(n-1)}{2 n+a(n+1)} & =\text { const, } \\
& k=1,2, \ldots, N .
\end{aligned}
$$

Therefore, based on the expression of the restored functions of two dynamic discrete GM (1,1) models, that is, (23) and (24), it is found that they are two homogeneous exponential functions.

Property 2 (isomorphic property). The restored functions of the concave dynamic discrete GM $(1,1)$ model and the convex dynamic discrete GM $(1,1)$ model are isomorphic to the discrete GM $(1,1)$ model.

Proof. Let $\sigma_{1}=e^{-a}, \sigma_{2}=(1-0.5 a) /(1+0.5 a), \sigma_{3}=(2 n-a(n+$ $1)) /(2 n+a(n-1))$, and $\sigma_{4}=(2 n-a(n-1)) /(2 n+a(n+1))$; we can get four specific restored functions as follows:

$$
\begin{aligned}
& \hat{x}^{(0)}(k)=\left(1-\sigma_{1}^{-1}\right)\left(x^{(0)}(1)-b a\right) \sigma_{1}^{k-1}, \\
& \hat{x}^{(0)}(k)=\left(1-\sigma_{2}^{-1}\right)\left(x^{(0)}(1)-b a\right) \sigma_{2}^{k-1}, \\
& \hat{x}^{(0)}(k)=\left(1-\sigma_{3}^{-1}\right)\left(x^{(0)}(1)-b a\right) \sigma_{3}^{k-1}, \\
& \hat{x}^{(0)}(k)=\left(1-\sigma_{4}^{-1}\right)\left(x^{(0)}(1)-b a\right) \sigma_{4}^{k-1} .
\end{aligned}
$$

Expanding $\sigma_{1}, \sigma_{2}, \sigma_{3}$, and $\sigma_{4}$ based on the Maclaurin formula, we can get

$$
\begin{aligned}
\sigma_{1} & =e^{-a} \\
& =1-a+\frac{a^{2}}{2 !}-\frac{a^{3}}{3 !}+\cdots+(-1)^{n} \frac{a^{n}}{n !}+o\left(a^{n}\right), \\
\sigma_{2} & =\frac{1-0.5 a}{1+0.5 a} \\
& =1-a+\frac{a^{2}}{2}-\frac{a^{3}}{2^{2}}+\cdots+(-1)^{n} \frac{a^{n}}{2^{n-1}}+o\left(a^{n}\right) .
\end{aligned}
$$

Therefore, when $a \rightarrow 0, \sigma_{1}$ and $\sigma_{2}$ are equal; when $a \rightarrow 0$ and $n \rightarrow \infty, \sigma_{1}, \sigma_{2}, \sigma_{3}$, and $\sigma_{4}$ are equal. In other words, the restored functions of two dynamic discrete GM $(1,1)$ models have the same structure as the restored function of the GM $(1,1)$ model and the discrete GM $(1,1)$ model.

Property 3 (convertible property). The concave and convex dynamic discrete GM $(1,1)$ models can be transformed into the discrete GM $(1,1)$ model.

Proof. If $n \rightarrow \infty$, then we have

$$
\begin{aligned}
\frac{-2 n a}{2 n-a(n-1)} & \longrightarrow \\
\frac{-2 a}{2-a} & =1-\frac{2+a}{2-a} ; \\
\frac{2 n-a(n-1)}{2 n+a(n+1)} & \longrightarrow \frac{2-a}{2+a}, \\
\frac{-2 n a}{2 n-a(n+1)} & \longrightarrow \\
\frac{-2 a}{2-a} & =1-\frac{2+a}{2-a} ; \\
\frac{2 n-a(n+1)}{2 n+a(n-1)} & \longrightarrow \frac{2-a}{2+a} .
\end{aligned}
$$

Thus, when $n \rightarrow \infty$, (17) and (18) can be transformed to the restored function of the discrete GM $(1,1)$ model (see $(7)$ ).

\section{Solution and Optimization of the Equal Division Number}

Definitions 9 and 10 give two complete dynamic discrete GM $(1,1)$ models but they raise a puzzle, that is, how to design a suitable equal division number $n$, which can be used to calculate the simulation values and the prediction values based on (19) and (22) with less error and bigger fitting and prediction accuracies. In order to do this, this paper proposes two new methods, namely, the gradually heuristics method and the dichotomy method, and uses them to calculate the optimal equal division number $n$.

\section{(1) The Gradually Heuristics Method}

Step 1. Based on (19) and (22), we can find that parameters $a$ and $b$ should be solved before the calculation of equal division number $n$. However, the background values $z^{(1)}(k)$ are required to obtain $a$ and $b$. Thus, the first step is the calculation of background values by $z^{(1)}(k)=\left(x^{(1)}(k)+\right.$ $\left.x^{(1)}(k-1)\right) / 2$, where $k=2,3, \ldots, n$.

Step 2. If the background values $z^{(1)}(k)$ are obtained, then we can calculate the parameter vector based on (5) by using the least square method. Then, the development coefficient $a$ and the grey action parameter $b$ can be got. 
Step 3. Analyze $\sigma_{k}=x^{(1)}(k+1) / x^{(1)}(k)$ and judge the characteristic of the 1-AGO sequence. If $\sigma_{k}$ is increasing, the 1-AGO sequence belongs to a concave function; if $\sigma_{k}$ is diminishing, the 1-AGO sequence belongs to a convex function.

Step 4. Construct the dynamic discrete GM $(1,1)$ model as follows. If the 1-AGO sequence is concave, we put $a$ and $b$ into (17) to construct the concave dynamic discrete GM $(1,1)$ model. Here, parameters $a$ and $b$ are calculated by Step 2. If the 1-AGO sequence is convex, we put $a$ and $b$ into (18), to construct the convex dynamic discrete GM $(1,1)$ model.

Step 5. Calculate the simulation values according to the following method. Take different values (starting with an appropriate value) to solve the discrete GM $(1,1)$ model with different equal division numbers $n$, and then obtain the corresponding simulated sequence $\widehat{X}_{n}^{(0)}(k)$.

Step 6. Calculate the average absolute error. This value is calculated as a percentage of the relative error generated from each actual data and its fitting value based on the formula $\bar{r}_{n}=\sum_{k=1}^{N}\left|\left(x^{(0)}(k)-\widehat{x}^{(0)}(k)\right) / x^{(0)}(k)\right| / N$.

Step 7. Determine the equal division number $n$ based on the following principle. If $\bar{r}_{n}$ is increasing, then $n$ is the equal division number, which is more suitable to the original sequence than the interval equal value. Then put it into (16) or (22); we can get the dynamic discrete GM $(1,1)$ model.

(2) The Dichotomy Method. Parameter $n$ solved by the gradual heuristics is an integer; however, the best equal division number is often not an integer; it can be further optimized. According to the calculation process of the gradual heuristics, we can find that there is only one extreme (the least error) in the vicinity of $n$. Thus, we can use the dichotomy method to further subdivide the integer $n$ to meet the accuracy requirements. The corresponding steps are described as follows.

Step 1. Calculate the equal interval as follows. Based on parameter $n$ obtained by the gradual heuristics method, we can extract the interval $[n-1, n+1]$ and then divide it into $[n-1, n]$ and $[n, n+1]$.

Step 2. Calculate the midpoint value of the average error. The midpoint values of the two intervals $n_{1}=((n-1)+n) / 2$ and $n_{2}=(n+(n+1)) / 2$ are substituted into the average absolute error formula separately to calculate the average absolute error rates.

Step 3. Examine the average errors. If $\bar{r}_{1}<\bar{r}_{n}<\bar{r}_{2}$ (or $\bar{r}_{2}<$ $\left.\bar{r}_{n}<\bar{r}_{1}\right)$, then the interval decreases to $[n-1, n]($ or $[n, n+1])$; if $\bar{r}_{n}<\bar{r}_{1}, \bar{r}_{n}<\bar{r}_{2}$, then the interval decreases to [( $(n-1)+$ $n) / 2,(n+(n+1)) / 2]$.

Step 4. Optimize the equal division number $n$. If the new interval is obtained under the conditions $\bar{r}_{n}<\bar{r}_{1}$ and $\bar{r}_{n}<\bar{r}_{2}$, the process from Step 2 to Step 4 is repeated. Otherwise, the new interval would be regarded as a dichotomy interval, and the process from Step 1 to Step 4 would need to be repeated until the required accuracy is obtained.

Thus, we provide two approaches to solve the equal division number, namely, the gradually heuristics method and the dichotomy method, which is a key parameter in the proposed dynamic discrete GM $(1,1)$ model. Of course, the corresponding optimization processes are given. On the basis of the aforementioned approaches, two new dynamic discrete GM $(1,1)$ models can be used to predict the simple sample. In the following section, we provide a practical case to demonstrate its effectiveness and superiority.

\section{Application of the Dynamic Discrete GM $(1,1)$ Model in Urbanization Conflict Events}

Urbanization is an important indicator of a country's development. In recent years, Chinese urbanization and economic development have made significant progress. However, Chinese urbanization also brought a series of contradictions and challenges. Among them, the conflict of urbanization has been intensified and the social contradictions have been highlighted. Tens of thousands of the group conflicts have taken place because of various social contradictions in China. Mass incidents caused by urbanization have increasingly become the focus of social attention and have become an important factor affecting the healthy development of China's economic. Social conflicts caused by the process of urbanization are the negative factors and could destroy the preexisting social stability. If they cannot be effectively resolved, the contradiction could have a greater detrimental effect and have a serious impact on China's urbanization process of project construction and social stability. Among them, the group conflict events caused by land requisition, demolition, and environmental problems of the urbanization in China constitute the majority of conflict events.

In this paper, we mainly focus on the study of land requisition, demolition, and environmental issues and fitting and forecasting the group conflict events in China's urbanization. As the Chinese statistical system is developed fairly late, there is a serious lack of relevant data. The policies often exhibit cyclical change and the data shows a periodic irregular trend. Thus, the grey prediction model based on the limited samples can effectively deal with the above problem. Therefore, this paper uses the fitting of the dynamic discrete GM $(1,1)$ models to predict the number of the urbanization conflict events in China and analyzes social problems that presently exist in China. Since the use of the proposed dynamic discrete GM $(1,1)$ models is consistent, this paper only uses the concave dynamic discrete GM $(1,1)$ model to predict the number of the urbanization conflict events, based on which the optimization of the dynamic discrete GM $(1,1)$ models is further analyzed.

5.1. Prediction of the Number of Urbanization Conflict Events in China. According to statistics from the Chinese Federation of Labour and the Blue Book of the Chinese Academy of Social Sciences, conflict events involving more than 30 people 
TABLE 1: Statistics of conflict events from 2008 to 2014 in China.

\begin{tabular}{lccccccc}
\hline Years & 2008 & 2009 & 2010 & 2011 & 2012 & 2013 & 2014 \\
\hline Pieces & 129 & 132 & 151 & 172 & 189 & 184 & 192
\end{tabular}

Data from the Chinese Federation of Labour and the Blue Book of the Chinese Academy of Social Sciences.

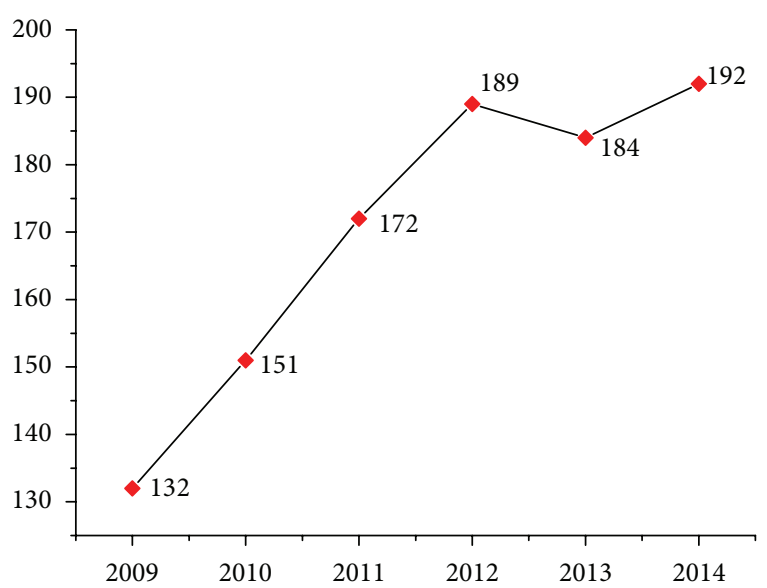

FIGURE 3: The original data of conflict events in 2008-2014 in China.

in the process of urbanization in 2008-2014 in China are shown in Table 1 and the data trend is depicted in Figure 3. Among them, the selection of the conflicts is based on two aspects: the land acquisition and the environment.

Using conflict events in $2008-2013$ as modeling data to simulate these six data points, we can predict the number of group conflict events that may occur in 2014. From the original data in Table 1, the number of group conflict events increased during 2008-2013, except 2013. This means that the marginal growth rate of the 1-AGO sequence is increased. Therefore, the concave dynamic discrete GM $(1,1)$ model is more suitable than the convex one. Thus, in this empirical example, the concave dynamic discrete GM $(1,1)$ model is selected and is used to make comparisons with other GM $(1,1)$ models.

According to $\widehat{\alpha}=\left(B^{T} B\right)^{-1} B^{T} Y$, we can get the development coefficient and grey action parameter of the GM $(1,1)$ model (GM model), the discrete GM $(1,1)$ model (DGM model), and the concave dynamic discrete GM $(1,1)$ model (EDDGM model) as follows: $-a=0.083$ and $b=122.96$. The development coefficient and grey action parameter of the optimal GM (1,1) model (OGM model) are $-a=0.057$ and $b=133.94$. These results are shown in Table 2 .

We further calculate the average relative error and prediction relative error of the four models, and the calculation results are shown in Tables 3 and 4 . Moreover, Figure 4 provides the one-to-one comparison between the fitting and actual values using four models. Figures 5 and 6 present the visual difference.

Based on Tables 2-4 and Figures 4-6, it can be found that the concave dynamic discrete GM $(1,1)$ model obtains best results as compared to the GM $(1,1)$ model, the discrete GM $(1,1)$ model, and the optimized GM $(1,1)$ model.
TABLE 2: Comparison between predicted and actual value of four models.

\begin{tabular}{lccccc}
\hline Years & $\begin{array}{c}\text { Actual } \\
\text { value }\end{array}$ & $\begin{array}{c}\text { DGM } \\
\text { model }\end{array}$ & $\begin{array}{c}\text { GM } \\
\text { model }\end{array}$ & $\begin{array}{c}\text { OGM } \\
\text { model }\end{array}$ & $\begin{array}{c}\text { EDDGM } \\
\text { model }\end{array}$ \\
\hline 2009 & 132 & 139.42 & 139.34 & 145.37 & 139.81 \\
2010 & 151 & 151.48 & 151.38 & 153.87 & 153.24 \\
2011 & 172 & 164.57 & 164.46 & 162.88 & 166.18 \\
2012 & 189 & 178.81 & 178.67 & 172.41 & 171.63 \\
2013 & 184 & 194.25 & 194.11 & 182.51 & 181.63 \\
2014 & 192 & 211.05 & 210.87 & 193.18 & 192.22 \\
\hline
\end{tabular}

TABLE 3: Average relative fitting errors of four models.

\begin{tabular}{lcccc}
\hline $\begin{array}{l}\text { Error } \\
\text { category }\end{array}$ & $\begin{array}{c}\text { DGM } \\
\text { model }\end{array}$ & $\begin{array}{c}\text { GM } \\
\text { model }\end{array}$ & $\begin{array}{c}\text { OGM } \\
\text { model }\end{array}$ & $\begin{array}{c}\text { EDDGM } \\
\text { model }\end{array}$ \\
\hline $\begin{array}{l}\text { Average } \\
\text { overall error } \\
(\%)\end{array}$ & 5.19 & 5.16 & 4.59 & 3.56 \\
\hline
\end{tabular}

TABLE 4: Relative prediction errors of four models.

\begin{tabular}{lcccc}
\hline Error category & $\begin{array}{c}\text { DGM } \\
\text { model }\end{array}$ & $\begin{array}{c}\text { GM } \\
\text { model }\end{array}$ & $\begin{array}{c}\text { OGM } \\
\text { model }\end{array}$ & $\begin{array}{c}\text { EDDGM } \\
\text { model }\end{array}$ \\
\hline $\begin{array}{l}\text { Predictive error } \\
(\%)\end{array}$ & 9.92 & 9.83 & 0.61 & 0.12 \\
\hline
\end{tabular}

5.2. Comparison and Analysis. Section 5.1 analyzes the fitting effect of conflict events in the process of urbanization in China based on several kinds of grey prediction models. Among them, the optimal result obtained by the concave dynamic discrete GM $(1,1)$ model is the best.

According to the results of the concave dynamic discrete GM $(1,1)$ model, it can be found that urbanization group conflict intensified day by day in China and social contradictions gradually increased. China's urbanization policy has presented many problems at the present stage and the government should take measures to solve these problems.

Thus, based on the fitting and predicted data, we provide some results and suggestions from the following three aspects.

(1) Constructing the Mechanism of Interest Integration. Through a variety of ways of achieving the expression of different levels of interest and consultation dialogue, we format a relatively stable and reasonable interest structure based on the special interests and values of different social strata in China. For reducing urbanization conflict and the maintenance of social fairness and justice, a balanced allocation of institutional arrangements should be carried out from the three aspects of society, market, and government.

(2) Improving Prevention and Disposal Mechanism. The government should establish the reasonable and effective mechanisms to resolve social contradictions and prevent the accumulation and intensification of social contradictions. Simultaneously, the government should improve the 


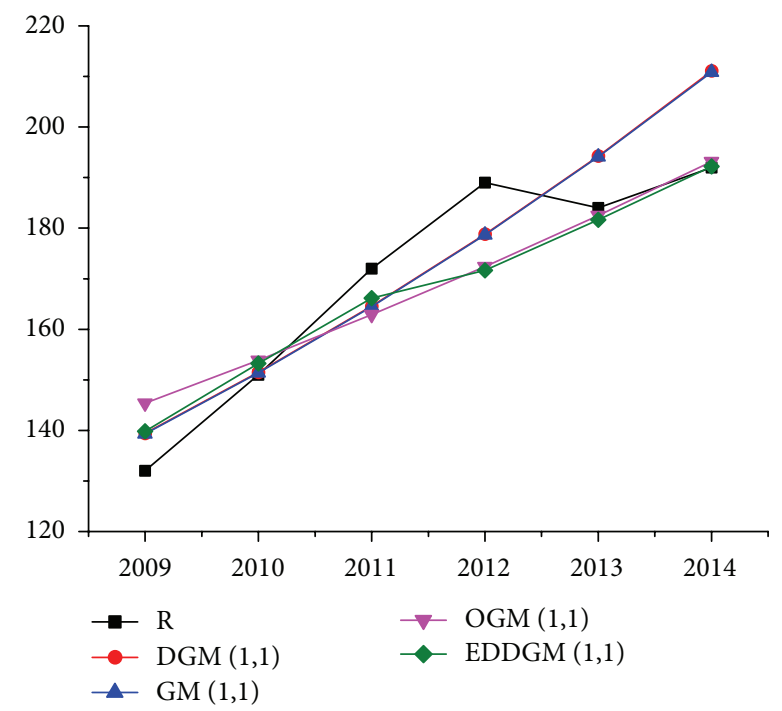

FIGURE 4: Comparison between the fitting and actual values using four models.

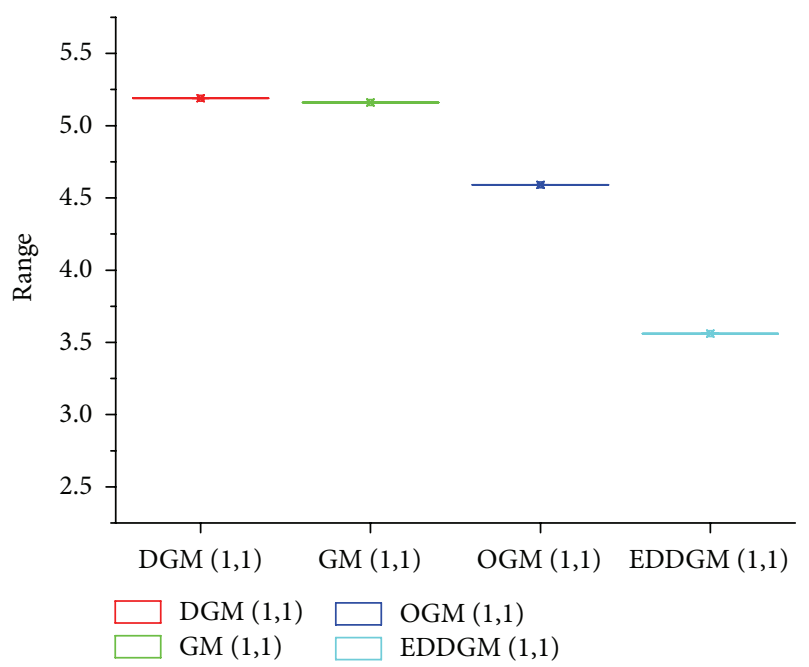

FIgURE 5: Average relative fitting errors of four models.

emergency response mechanism and purposefully guide the public.

(3) Participating in Collaborative Governance. The government should make full use of the supervisory function of public media in urbanization conflict management, enhance the function of informal organization to reflect demand, coordinate communication in the conflict, and foster the trust and tolerance of social capital in the governance of group incidents.

\section{Conclusions}

Urbanization conflict is an important issue in China and needs to be solved as soon as possible. It is a reflection of the contradiction of interests, which exposes the serious shortage of Chinese local governments and organizations in

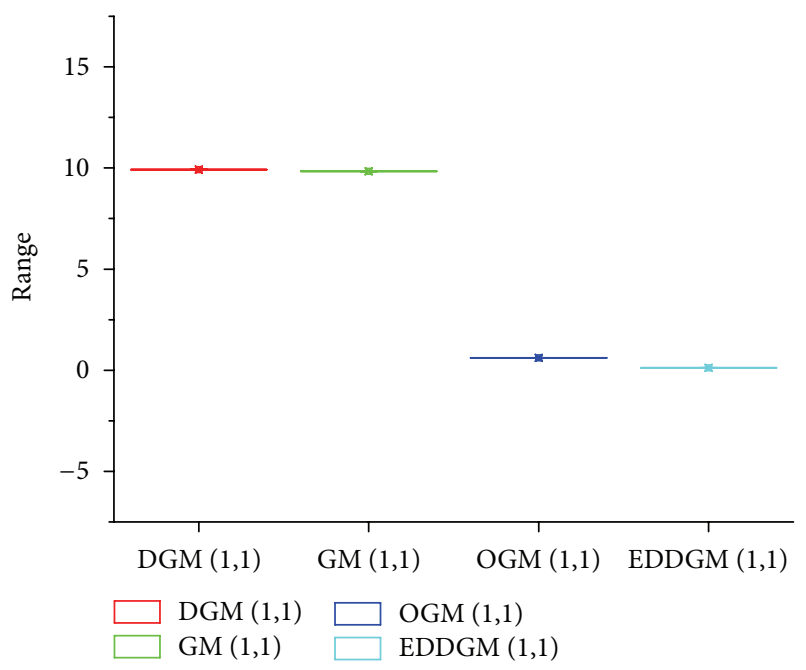

FIGURE 6: Prediction relative errors of four models.

management and decision-making. As the data is lacking and changes periodically, this paper has proposed the dynamic discrete GM (1,1) model based on the discrete GM $(1,1)$ model and an improved equal division number. Two optimal dynamic discrete GM $(1,1)$ models are obtained based on the gradual heuristic method and the dichotomy method. According to the characters of the dynamic discrete GM $(1,1)$ models, we have selected the urbanization conflict events in China as the object for prediction. Empirical results show that the rapid process of urbanization aggravates social conflicts in China. Finally, according to the problems exposed in the urbanization process in China, we have provided three suggestions for the promotion of urbanization construction in China. The dynamic discrete GM $(1,1)$ models proposed in this paper can be used to solve the problem of the periodic irregular change in the sequence better than the GM $(1,1)$ model, the optimal GM $(1,1)$ model, and discrete GM $(1,1)$ model.

\section{Competing Interests}

The authors declare that there is no conflict of interests regarding the publication of this paper.

\section{Acknowledgments}

This work was supported by the Natural Science Foundation of China (no. 71463063).

\section{References}

[1] D. Ju-Long, "Control problem of grey systems," Systems \& Control Letters, vol. 1, no. 5, pp. 288-294, 1982.

[2] S. F. Liu, Y. G. Dang, and Z. G. Fang, Grey System Theory and Its Application, Science Press, Beijing, China, 2004.

[3] N.-M. Xie and S.-F. Liu, "Discrete GM $(1,1)$ and mechanism of grey forecasting model," System Engineering Theory and Practice, vol. 25, no. 1, pp. 93-99, 2005. 
[4] N.-M. Xie and S.-F. Liu, "Research on extension of discrete grey model and its optimize formula," System Engineering Theory and Practice, vol. 26, no. 6, pp. 108-112, 2006.

[5] T. X. Yao and S. F. Liu, "Improvement of a forecasting discrete GM(1,1)," Systems Engineering, vol. 25, no. 9, pp. 103-106, 2007.

[6] Z.-X. Wang, Y.-G. Dang, and S.-F. Liu, "An optimal GM(1,1) based on the discrete function with exponential law," Systems Engineering-Theory \& Practice, vol. 28, no. 2, pp. 61-67, 2008.

[7] T. X. Yao, S. F. Liu, and N. M. Xie, "Study on the properties of new information discrete GM $(1,1)$ model," Journal of Systems Engineering, vol. 25, no. 2, pp. 164-170, 2010.

[8] W. Zhou, Z.-G. Fang, and S.-F. Liu, "Generalized GM(1, 1) forecast model based on the optimized level ratio," System Engineering Theory and Practice, vol. 30, no. 8, pp. 1433-1438, 2010.

[9] E. Kayacan, B. Ulutas, and O. Kaynak, "Grey system theorybased models in time series prediction," Expert Systems with Applications, vol. 37, no. 2, pp. 1784-1789, 2010.

[10] D.-C. Li, C.-J. Chang, C.-C. Chen, and W.-C. Chen, "A greybased fitting coefficient to build a hybrid forecasting model for small data sets," Applied Mathematical Modelling, vol. 36, no. 10, pp. 5101-5108, 2012.

[11] W. Zhou and J.-M. He, "Generalized GM $(1,1)$ model and its application in forecasting of fuel production," Applied Mathematical Modelling, vol. 37, no. 9, pp. 6234-6243, 2013.

[12] G. F. Liu, S. B. Huang, X. F. Piao, and Y. Cheng, "Hybrid prediction based on BP neural network and Markov Chain," in Proceedings of the 3rd International Conference on Control Automation and Systems Engineering, pp. 92-96, 2013.

[13] B.-H. Yang and J.-S. Zhao, "Fractional order discrete grey GM(1, 1) power model and its application," Control and Decision, vol. 30, no. 7, pp. 1264-1268, 2015.

[14] W. Zhou and D. M. Zhang, "An improved metabolism grey model for predicting small samples with a singular datum and its application to sulfur dioxide emissions in China," Discrete Dynamics in Nature and Society, vol. 2016, Article ID 1045057, 11 pages, 2016.

[15] A. Portes and B. R. Roberts, "The free-market city: latin American urbanization in the years of the neoliberal experiment," Studies in Comparative International Development, vol. 40, no. 1, pp. 43-82, 2005.

[16] A. T. W. Yu, Y. Wu, B. Zheng, X. Zhang, and L. Shen, "Identifying risk factors of urban-rural conflict in urbanization: a case of China," Habitat International, vol. 44, pp. 177-185, 2014.

[17] A. T. W. Yu, Y. Wu, J. Shen, X. Zhang, L. Shen, and L. Shan, "The key causes of urban-rural conflict in China," Habitat International, vol. 49, pp. 65-73, 2015.

[18] D. Zhou, J. Xu, L. Wang, and Z. Lin, "Assessing urbanization quality using structure and function analyses: a case study of the urban agglomeration around Hangzhou Bay (UAHB), China," Habitat International, vol. 49, pp. 165-176, 2015.

[19] C.-W. Su, T.-Y. Liu, H.-L. Chang, and X.-Z. Jiang, "Is urbanization narrowing the urban-rural income gap? A cross-regional study of China," Habitat International, vol. 48, pp. 79-86, 2015.

[20] Y. Wang, Y. Liu, Y. Li, and T. Li, "The spatio-temporal patterns of urban-rural development transformation in China since 1990," Habitat International, vol. 53, pp. 178-187, 2016.

[21] G. J. Tan, "The structure method and application of background value in grey system GM $(1,1)$ model (I)," Systems EngineeringTheory \& Practice, vol. 20, no. 4, pp. 98-103, 2000.
[22] D. Luo, S. F. Liu, and Y. G. Dang, "The optimization of grey GM (1,1) model," China Engineering Science, vol. 5, no. 8, pp. 50-54, 2003.

[23] L. Liu, H. G. Wang, and B. W. Wang, "The optimization of grey GM $(1,1)$ model based on background level," Statistics and Decision, vol. 277, no. 1, pp. 153-155, 2009.

[24] W. Tang and C. Xiang, "Quadratic interpolation based on improved GM $(1,1)$ model prediction methods," Chinese Journal of Management Science, vol. 14, no. 6, pp. 109-112, 2006.

[25] X. Wang and S. Yang, "The improvements and applications of forecasting method," in GM, vol. 1, 1, pp. 129-134, 2012. 


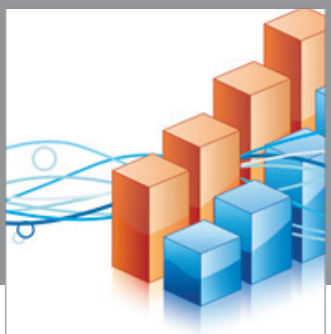

Advances in

Operations Research

vatem alat4

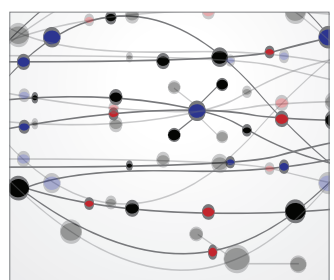

\section{The Scientific} World Journal
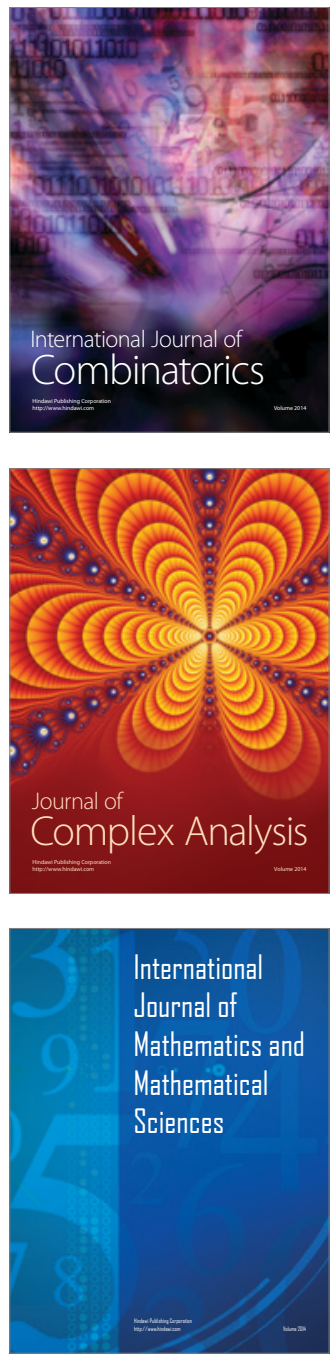
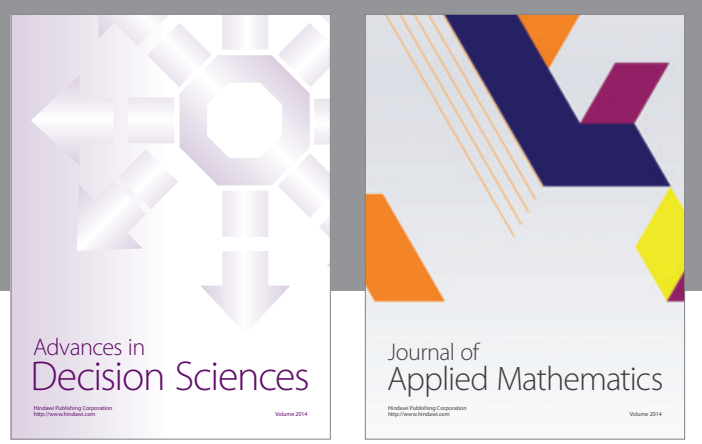

Algebra

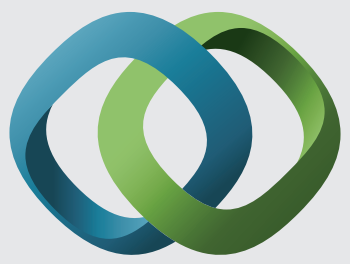

\section{Hindawi}

Submit your manuscripts at

http://www.hindawi.com
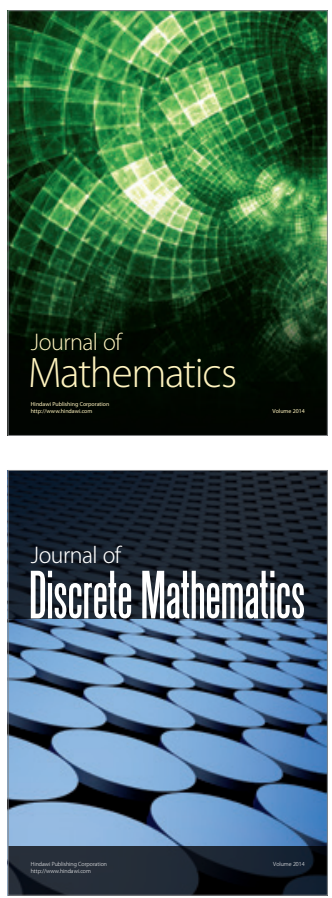

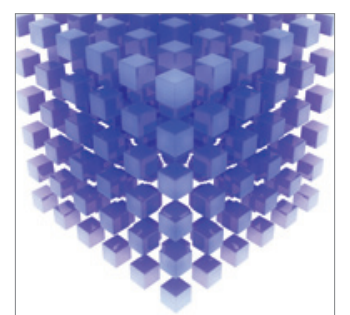

Mathematical Problems in Engineering
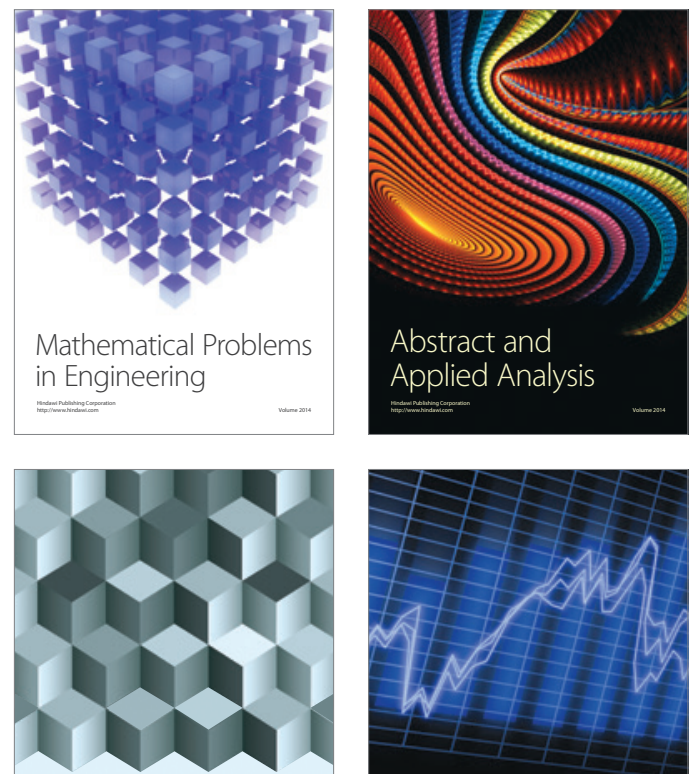

Journal of

Function Spaces

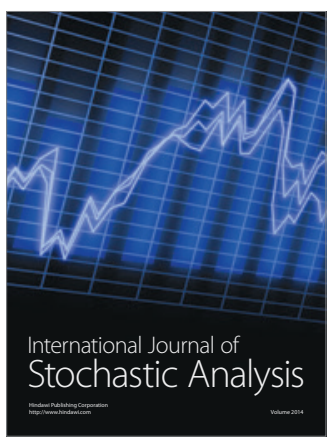

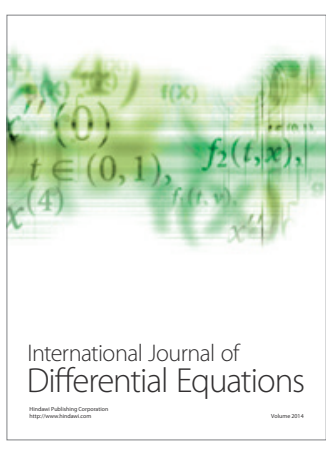
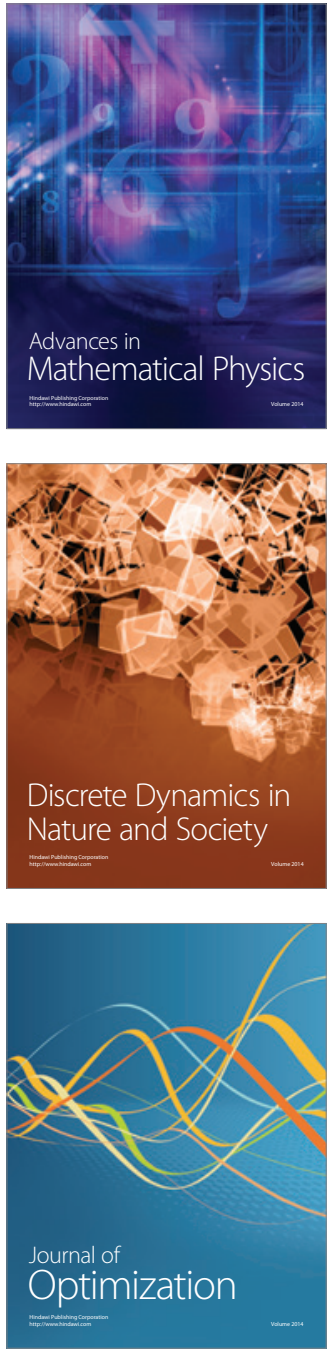